\title{
Vorticity dissipation associated with vortex pairing within a curved shear layer
}

\author{
Hiroyuki HANIU*, Kazutoyo YONEYAMA**, Mohammad Rofiqul ISLAM***, Kazunori TAKAI*, \\ Yoshihiro OBATA* and Mohammad Emdadul HAQUE*** \\ *Department of Mechanical Engineering, Kitami Institute of Technology \\ 165 Koen-cho, Kitami, Hokkaido 090-8507, Japan \\ E-mail: harry@mail.kitami-it.ac.jp \\ **Toshiba Corporation, Suehiro-cho, Tsurumi-ku, Yokohama,230-0045, Japan \\ ***Department of Mechanical Engineering, Rajshahi University of Engineering \& Technology, Rajshahi 6204, Bangladesh
}

Received: 9 December 2016; Revised: 15 March 2017; Accepted: 19 April 2017

\begin{abstract}
In this study, vorticity dissipation phenomenon within a curved shear layer while Karman vortices are formed behind a body was investigated experimentally based on time variation of energy dissipation function distribution. The vorticity dissipation within a separated shear layer would be caused by viscous effect associated with shear strain in the process of pairing between two vortices formed by Kelvin-Helmholtz instability. Therefore, in this experiment, the vortex pairing phenomenon within a separated shear layer was reproduced within a centrifugally stable curved free shear layer. Time variation of velocity distribution in one period of vortex pairing process was obtained by means of L.D.V. and phase ensemble averaging technique. By differentiating the velocity distribution for each phase of pairing process, time varying distributions of vorticity, shear strain rate and energy dissipation function were obtained and investigated. As the results, it was found that the strong shear strain occurs and energy is dissipated significantly when the vortices are started to starch in the process of pairing. Also, it was found that the diffusion of vorticity from the vortices to surrounding irrotational fluid becomes significant after the vortices are fully stretched, instead of in the process of stretching. Moreover, it was found that the energy dissipation by shear strain is almost ended when the pairing vortices align vertically, that is defined as the "merging location."
\end{abstract}

Key words : Vorticity dissipation, Vortex pairing, Curved shear layer, Vorticity, Shear strain rate, Energy dissipation function, LDV, Phase ensemble averaging technique

\section{Introduction}

Karman vortices are generated behind a bluff body in a flow, and fluctuating fluid forces act on the body. Previous research groups Gerrard (1966) and, Sarpkaya and Schoaff et al. (1979) found that the circulation of a Karman vortex which determines the magnitude of the fluctuating fluid force is closely related to the entrainment and dissipation of the vorticity in a separated shear lasyer. In an experiment of Karman vortex formation using old fashioned particle trace method, Mair and Maull et al. (1971) reported that about $15 \%$ of the circulation of one side of the separated shear layer is cancelled by the circulation of opposite sign entrained from the other side of the separated shear layer, and about $20 \%$ is dissipated by entrainment into the other side of the shear layer and residence in the flow circulating region. However, the analysis of the old fashioned particle trace method is based on the assumption that a fluid particle moves with constant vorticity of its initial value, that is ignoring diffusion of voriticity to the surrounding fluids, and brought very rough estimate of the lost circulation. Miyakoshi and Haniu et al. (2010) studied with pairing of small instable vortices in a separated shear layer at very vicinity of separation point from a body, and found that the shear strain based on viscous interaction associated with the pairing would be one of the cause of vorticity dissipation. However, there is almost no literature regarding the relation between shear strain and vorticity dissipation in the process of vortex pairing. No detailed report on the vortex pairing phenomena in a separated shear 
layer with stream line curvature formed right down stream of the separation point is available. Therefore, it is worthwhile to clarify the vorticity dissipation phenomena in a curved shear layer not only for formation mechanism and control of the large scale vortex (Karman vortex) but also for providing benchmark data for numerical simulations.

Previous research group Winant and Browand (1974) reported that the vortices are elongated along the flow direction during the process of pairing in free shear layer. Another research group Browand and Weidman (1976) found that significant Reynolds-stress production is associated with pairing interaction. In the pairing process, Aref and Siggia (1980) proposed that vortex fluid will get drawn into long, thin filaments, with viscosity, viscous effects will be dominated in the filaments, and enstrophy will be destroyed. Ho and Huang (1982) reported that vortices align vertically where sub-harmonics reach its peak and they defined such a place as "merging location". Metcalfe et al. (1987) reported that Reynolds stress is positive during early stage of pairing and later it becomes negative, while positive Reynolds stress feeds energy from mean flow into perturbation field and negative Reynolds stress feeds energy from fluctuation into mean flow. All above reports except for Miyakoshi and Haniu et al. (2010) dealt with straight mixing layers, and report dealt with curved free mixing layer such as separated shear layer from a body is scant. Also, report dealt with vorticity dissipation associated with vortex pairing is scant. According to the study by Haniu and Ramaprian (1989) on curved jets, the separated shear layer in the vicinity of separation point is in centrifugally stable state due to combination of velocity gradient and stream line curvature. In a separated shear layer, small Kelvin- Helmholtz instability vortices generating one after another are repeatedly merged under influence of suitable centrifugally stable flow field and clustered into a large scale vortex (Karman vortex).

In this study, as a quasi-steady- state model of separated shear layer, centrifugally stable curved free shear layer have been provided, and one cycle of velocity distribution data in pairing process of small vortices formed within the shear layer, which are similar to the Kelvin-Helmholtz instability vortices, were acquired by means of L.D.V. and phase ensemble averaging technique. Distributions of vorticity, shear strain rate and energy dissipation function were calculated by numerical differentiation of phase ensemble averaged velocity distribution data for each phase. From relation among those distributions, vorticity dissipation in the process of vortex pairing was investigated in the point of view of energy dissipation.

\section{Nomenclatures}

$\boldsymbol{A}_{1}$ : amplitude of primary oscillating frequency $f_{\mathrm{v}}$

$\boldsymbol{A}_{2}$ : amplitude of sub harmonic oscillating frequency $f_{\mathrm{v}} / 2$

$\boldsymbol{A}_{\mathrm{H}}$ : higher displacement amplitude of splitter plate trailing edge

$\boldsymbol{A}_{\mathrm{L}}$ : lower displacement amplitude of splitter plate trailing edge

$\boldsymbol{d}$ : scale of vortices

div: divergence of flow

$f_{v}$ : time averaged formation frequency of Kelvin-Helmholtz instability vortices

$\boldsymbol{R}$ : radius of curvature

$\boldsymbol{R}_{\mathrm{e}}$ : Reynolds number based on scale of vortices $d$ and free stream velocity $U_{0}$

$\boldsymbol{t}$ : elapse of time

$\boldsymbol{u}$ : velocity in $x$ coordinate

$\boldsymbol{U}_{0}$ : free stream velocity

$v$ : velocity in $y$ coordinate

$\boldsymbol{x}$ : straight coordinate along flow direction at the exit of flow contraction

$\boldsymbol{y}$ : straight coordinate perpendicular to $x$ coordinate

$\boldsymbol{y}(t)$ : time variation of splitter plate trailing edge displacement in $y$ direction

$\varepsilon$ : shear strain rate

$\Gamma$ : circulation

$\theta$ : phase of time varying flow field

$\Phi$ : dissipation function

$\omega$ : vorticity

$\omega_{\max }:$ maximum vorticity 


\section{Experimental Apparatus and Methods}

The water flow facility for the presented study as shown in Fig.3.1 was constructed in a water tank with flow settling chamber, flow contraction and curved wall. Water was recirculated by a submerged pump placed in the downstream end. Depth of water was set to $400 \mathrm{~mm}$ and measuring plane was located horizontally $200 \mathrm{~mm}$ above the bottom floor of the water tank. Inside the settling chamber, two acrylic porous walls were placed in parallel to reduce the flow disturbance caused by water supply from the pump through a pipe, and honeycomb was placed in the downstream to straighten the flow before the contraction section of ratio 2:1. Two straight walls of 330mm long in parallel with $300 \mathrm{~mm}$ apart were attached after the contraction. At the inlet part of the straight walls, another honeycomb was placed for flow uniformity. Main stream velocity at the middle between the ends of two parallel walls was set to $6 \mathrm{~cm} / \mathrm{sec}$, and water temperature was maintained within a range of $20 \pm 2{ }^{\circ} \mathrm{C}$. Dye injection at the middle of the main stream near the downstream end of the straight walls show smooth steak line without spread of dye with slow and very small wavy motion. This indicates the main stream is laminar flow. L.D.V. measurement made slightly downstream yielded fluctuation level in terms of RMS value of velocity in stream wise component was approximately

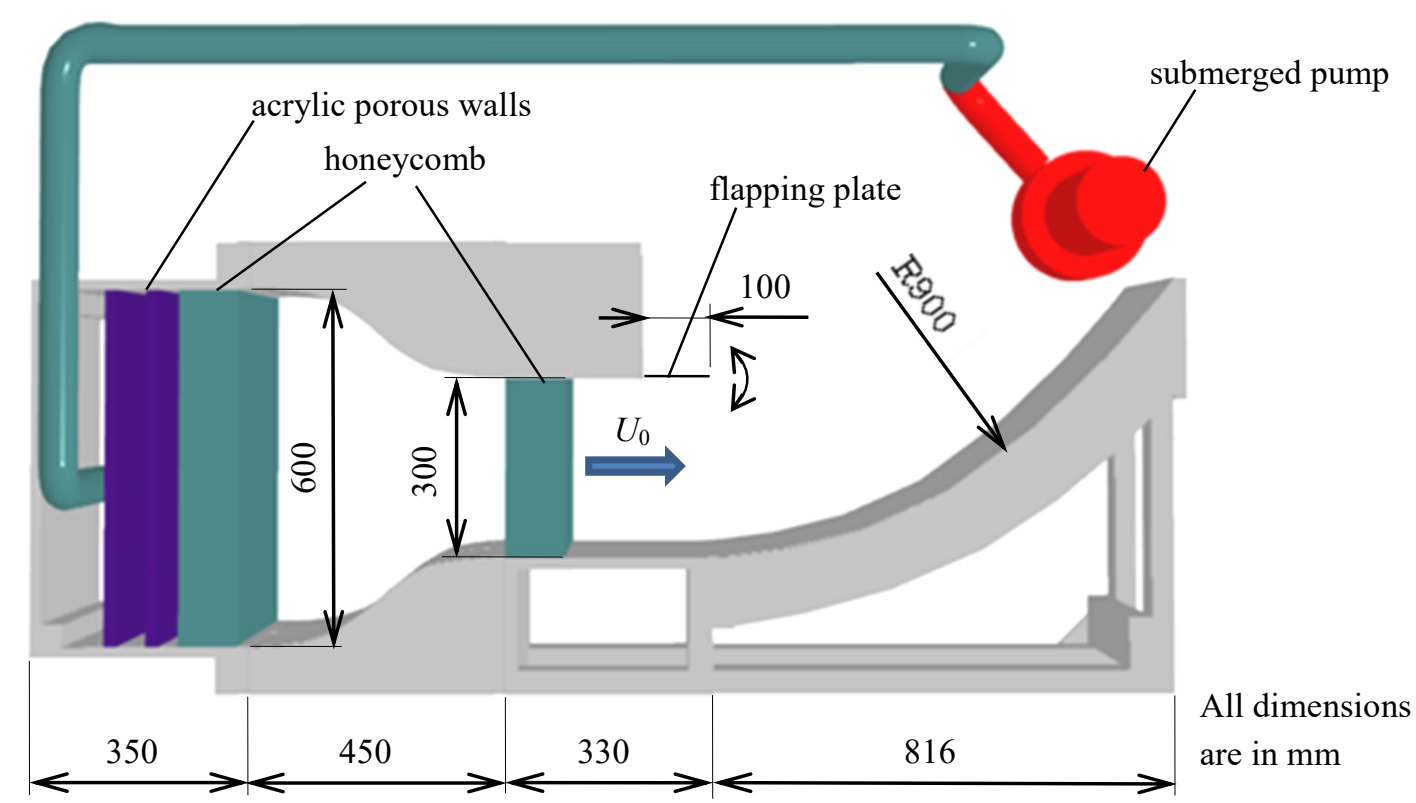

Fig. 3.1 Schematic view of the water flow facility with its dimensions

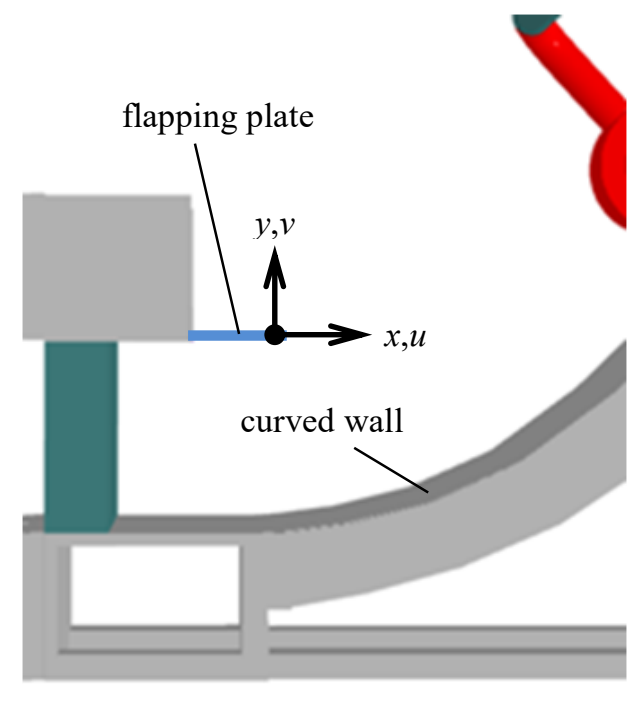

Fig. 3.2 Coordinate system 
$3.5 \%$ of the main steam velocity. Since the RMS value contains tracking noise of L.D.V. signal processer, actual velocity fluctuation level well be lower. After one of the straight walls, curved wall with radius $R$ of $900 \mathrm{~mm}$ was connected in order to give the main stream line coverture. A $100 \mathrm{~mm}$ long thin aluminum flapping plate was attached by hinges at downstream end of another wall. This side of the wall is located at inner portion of main stream line curvature. As shown in Fig.3.2, the coordinate system has origin at trailing edge of the flapping plate when the plate is aligned with the solid straight wall. The coordinate aligned with the straight wall is defined as $x$ axis and its velocity component is defined as $u$. The coordinate perpendicular to the $x$ axis and orienting inner side of the stream line curvature is defined as $y$ and its velocity component is defined as $v$.

In the downstream of the flapping plate trailing edge, a curved mixing layer is formed and its flow field is in centrifugally stable state, because the main stream velocity increases as it goes toward outer side of the curvature. In the mixing layer, unstable vortices are produced by Kelvin-Helmholtz instability. Then the neighboring vortices interact each other with their induced velocity and push away each other toward opposite side from their original flowing pass. However, the centrifugally stable state nicely prevents from that happen, and vortices are repeatedly merged in the downstream. Therefore, the flow phenomena occurring in a separated shear layer from a bluff body can be reproduced in this flow facility. As reported by Haniu and Ramaprian (1989), the centrifugally stable state is known to reduce turbulent intensity. In this study, it will influence only on the flowing pass of the vortices, but will not influence on the distributions of vorticity, shear strain rate and energy dissipation. There are no reports dealing with the influence of stream line curvature on those distributions. On the other hand, due to presence of irregularity in the natural formation of the instable vortices, jitter is occurring in the measured velocity signal, and not only it causes some deformation in the time variation of velocity data obtained by phase ensemble averaging technique, but also it makes difficulty to obtain a reference signal for the phase ensemble averaging. Therefore, in this experiment, periodicity of the vortices generation was enhanced by oscillating the flapping plate at time mean frequency of the Kelvin-Helmholtz instable vortices formation. Also, reference single for the phase ensemble averaging was obtained by attaching a photo sensor to the oscillation mechanism. The flapping plate was oscillated with the act of link mechanism using a high torque servo for models which was controlled by PWM signal from a micro-computer. Oscillating frequency $f_{v}$ of the flapping plate was set to $1.02 \mathrm{~Hz}$ which is time averaged natural frequency of the Kelvin-Helmholtz instable vortices formation. Trailing edge of the flapping plate was displaced with time as shown in Fig.3.3 according to Eq.3.1. With this control, larger and smaller displacements appear alternately to place vortices alternately on the inner and outer stream lines with respect to the stream line curvature and periodicity of vortex pairing was enhanced. From visualization study, coefficient $A_{1}$ and $A_{2}$ in Eq.3.1 were adjusted to make the probability of vortex pairing over $90 \%$. As the results, displacements $A_{\mathrm{H}}$ and $A_{\mathrm{L}}$ in Fig.3.3 were set to $4.0 \mathrm{~mm}$ and $2.8 \mathrm{~mm}$ respectively. The reference signal for phase ensemble averaging was popped up when the trailing edge displacement becomes largest value of $A_{\mathrm{H}}$ to fix the phase difference between the reference signal and vortices pairing.

$$
y(t)=-A_{1} \cos \left(2 \pi f_{v} t\right)-A_{2} \cos \left(\pi f_{v} t\right)-\left(8 A_{1}^{2}+A_{2}^{2}\right) /\left(8 A_{1}\right)
$$

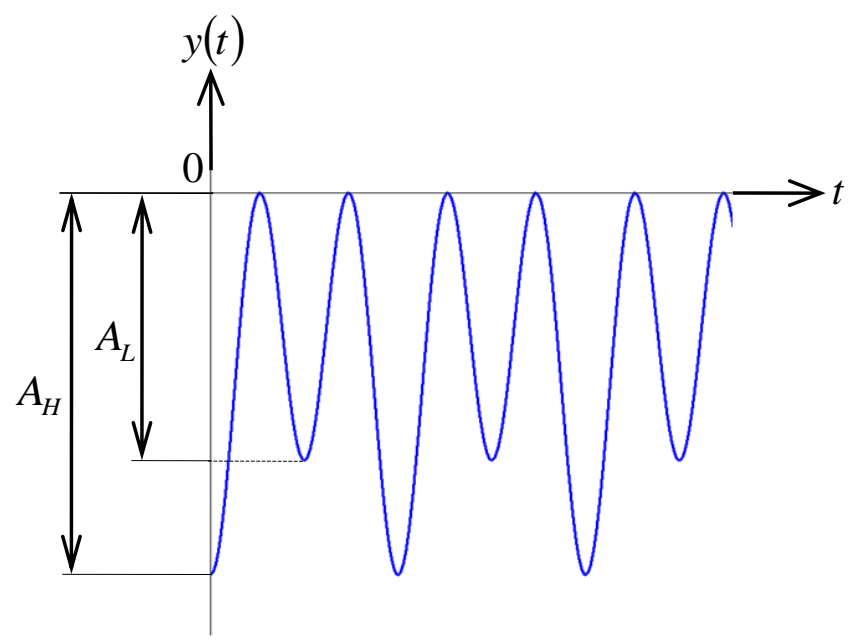

Fig. 3.3 Displacement of the flapping plate's trailing edge with time 
Wignanski et al. (1979) reported that a two-dimensional coherent eddy preserves in spite of strong disturbance and two-dimensional forcing of mixing layer improves coherence and repeatability of the large eddy structure. In this study, since size of the vortices is not small compare to the shear layer thickness, vortices can be treated as large eddies and similar effect of two-dimensional forcing will be there.

For the flow visualization, a thin translucent plastic sheet with radial lines and circular arcs for back ground scale was placed on the bottom floor of the test section. The sheet was illuminated from underneath of the bottom floor, and black dye was injected from the trailing edge of the flapping plate to show the formation of the vortices. Time varying images of the vortices were taken from top of the test section by a video camera. Flow visualization images taken at $0.4 \mathrm{sec}$ interval with the above mentioned oscillating condition of the flapping plate are presented in Fig.3.4. From (a) to (f) of the figures, it is seen that the vortex denoted by B is passing the leading vortex denoted by A while they are flowing downstream, and they are starting to merge at approximately $x=50 \mathrm{~mm}$. From the fact that the interval of the background radial lines along the arc on which vortices are tracing is approximately $50 \mathrm{~mm}$, size $d$ of each vortex can be read as 20 to $30 \mathrm{~mm}$. Therefore, based on the characteristic size $d$ of the vortices and characteristic velocity $U_{0}$ of the main stream, Reynold number $R$ e is calculated as 1,200 to 1,800 . This value is not very small in contrast to the value of Karman vortex.

A glass plate window is fitted on the bottom floor of the water tank through which velocity was measured by fiber optic L.D.V. system with reverse flow measurement capability by means of dual frequency shifters. Entire L.D.V. system was mounted on a traverse system located underneath of the glass window on the water tank floor. Ranges of velocity measurement by means of L.D.V. are $30 \mathrm{~mm}$ to $130 \mathrm{~mm}$ at $2.5 \mathrm{~mm}$ interval in $x$ coordinate and $-13 \mathrm{~mm}$ to $43 \mathrm{~mm}$ at $1.5 \mathrm{~mm}$ interval in $y$ coordinate. In a shear layer, since the velocity gradient in $y$ direction is larger than that in $x$ direction, higher resolution of space is required in $y$ direction. When vorticity and shear strain rate is calculated by numerical differentiation, the point of interest require few data points at both left and right side for derivative in $x$ direction and similarly at both top and bottom side for derivative in $y$ direction. Therefore, viewing ranges of vorticity and shear strain rate became 35 to $125 \mathrm{~mm}$ in $x$ coordinate and -10 to $40 \mathrm{~mm}$ in $y$ coordinate. Since a laser beam of L.D.V. hits flapping plate in the region of $x<30 \mathrm{~mm}$, flow measurements could not be done in very near region of the flapping plate. In L.D.V. measurements for ensemble averaging, sampling frequency was set to $200 \mathrm{~Hz}$ for both $u$ and $v$ components. Since the periodicity of vortex pairing is approximately 2 second, 400 velocity data are sampled in one pairing, and this yields about 0.9 degree of phase resolution. Anti-aliasing analogue low pass filter was set to Bessel mode to minimize phase shift. Also for further suppression of phase shift with analogue filter, according to characteristic chart of the filter, cutoff frequency was set to $20 \mathrm{~Hz}$ which is about 20 times of the vortex formation frequency of $1.02 \mathrm{~Hz}$. Sampling time duration of velocity data at each measuring point was 420 second which corresponds to about 200 times of vortex pairing and 1,167,000 data points. Phase reference signal from photo sensor attached to the flapping plate oscillation mechanism was also sampled simultaneously with the velocity data.

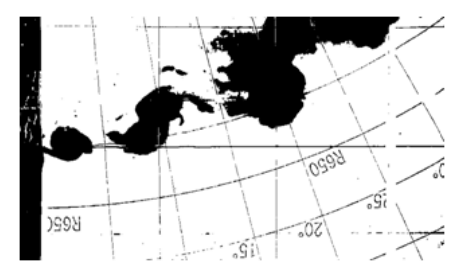

(a)

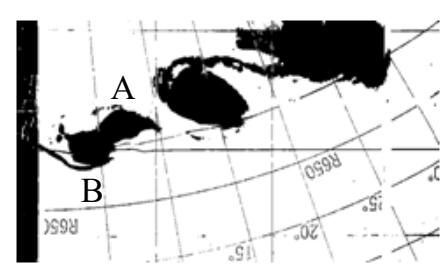

(d)

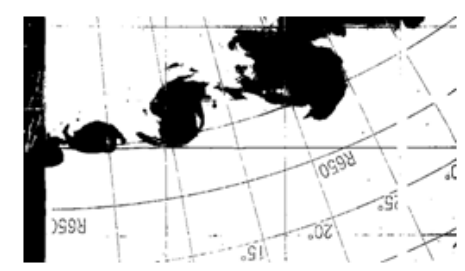

(b)

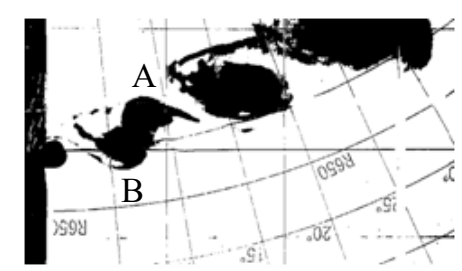

(e)

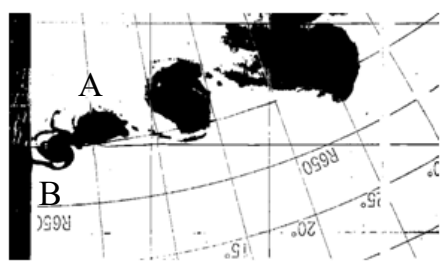

(c)

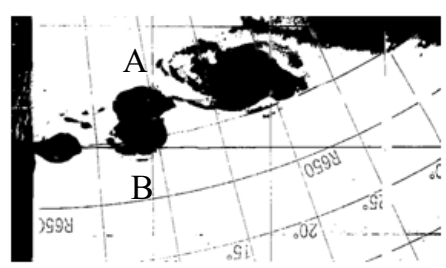

(f)

Fig. 3.4 Time series visualization images showing the size and pairing of vortices 
At each measuring point, one period of vortex pairing was equally divided into 60 phase angles of $\theta$, and data of closest time to each $\theta$ were repeatedly extracted and averaged to perform phase ensemble averaging. After completion of this process for every measuring point, ensemble averaged data for each phase was regrouped to form instantaneous velocity distributions. Then the velocity distribution for each phase was numerically differentiated to obtain instantaneous distributions of vorticity, shear strain rate and later mentioning dissipation function of kinematic energy. The vorticity is given in Eq. (3.2) and the shear strain rate is given in Eq. (3.3). For the numerical differentiation of velocity data, Savitzky-Golay method (1964) with five extraction data points and a cubic (point symmetry) weight function was attributed.

$$
\begin{aligned}
& \omega(x, y, \theta)=\frac{\partial v(x, y, \theta)}{\partial x}-\frac{\partial u(x, y, \theta)}{\partial y} \\
& \varepsilon(x, y, \theta)=\frac{\partial v(x, y, \theta)}{\partial x}+\frac{\partial u(x, y, \theta)}{\partial y}
\end{aligned}
$$

In an incompressible flow like this study, divergence given in Eq. (3.4) is supposed to be zero. In this study, velocity data were retaken for the measuring points where absolute value of the divergence is larger than 1.5 times of the standard deviation of the entire divergences to ensure the quality of the measurement. As the results, deviation of the divergence was kept within $1.9 \mathrm{~s}^{-1}$ which is $8.5 \%$ of the maximum vorticity in the entire measuring region.

$$
\operatorname{div}(x, y, \theta)=\frac{\partial u(x, y, \theta)}{\partial x}+\frac{\partial v(x, y, \theta)}{\partial y}=0
$$

Since a separated shear layer grows as it entrains surrounding irrotational flow, flow field is considered to be irrotational except for nearby a vortex. Vorticity is produced in the boundary layer along the contraction wall and the flapping plate surfaces, then released in to the separated shear in a form of thin sheet and it repeatedly roll up to form vortices. Kinematic energy dissipation of a flow is caused by stretching and shear strain. In the separated shear layer, the dissipating kinematic energy is considered to be the rotational energy of vortices. Especially in the process of the vortex pairing, energy dissipation due to strong shear strain associated with the interaction between vortices will be dominant. Kinematic energy dissipation due to viscosity can be expressed as dissipation function $\Phi$ defined in Eq. (3.5) presented by White (1974). Phase ensemble averaged velocity distribution for each phase obtained from velocity signal by means of L.D.V. was numerically differentiated to yield dissipation function distributions. From the equation, $\Phi$ is composed of stretching rate and shear strain rate, and it is seen that the rotational motion of the fluid is not directly related to $\Phi$. This implies that kinematic energy is considered to be dissipated by stretching and shear strain associated with interaction between two rotating vortices.

$$
\Phi(x, y, \theta)=\mu\left[2\left(\frac{\partial u(x, y, \theta)}{\partial x}\right)^{2}+2\left(\frac{\partial v(x, y, \theta)}{\partial y}\right)^{2}+\left(\frac{\partial v(x, y, \theta)}{\partial x}+\frac{\partial u(x, y, \theta)}{\partial y}\right)^{2}\right]
$$

\section{Results and Discussions}

\subsection{Variations of circulation and maximum vorticity}

Variation in $x$ direction of circulation $\Gamma$ through cross sections perpendicular to $x$ axis in one period of vortex pairing is presented in Fig.4.1. In the calculation of circulation, product of $x$ component velocity $u(x, y, \theta)$ and vorticity $\omega(x, y, \theta)$ was numerically doubly integrated with respect to $y$ and $\theta$. Even though the data are somewhat scattered due to error in velocity measurements and numerical differentiation, circulation rapidly decreased from around $x=45 \mathrm{~mm}$ to $55 \mathrm{~mm}$ and stays nearly constant in the downstream. Therefore, vorticity is considered to be dissipated significantly in the range around $x=45$ to $55 \mathrm{~mm}$.

Variation in $x$ direction of the maximum vorticity $\omega_{\max }$ is presented in Fig.4.2. The maximum vorticity gradually decreases from about $x=40 \mathrm{~mm}$ to $x=60 \mathrm{~mm}$, then it decreases rapidly toward about $x=80 \mathrm{~mm}$ and becomes as small as one third of the value at about $x=40 \mathrm{~mm}$. This implies that vorticity diffusion from vortices to surrounding fluid is moderate in the highly interacting stage of vortex pairing, and the diffusion becomes significant in the weakly interacting latter stage. 


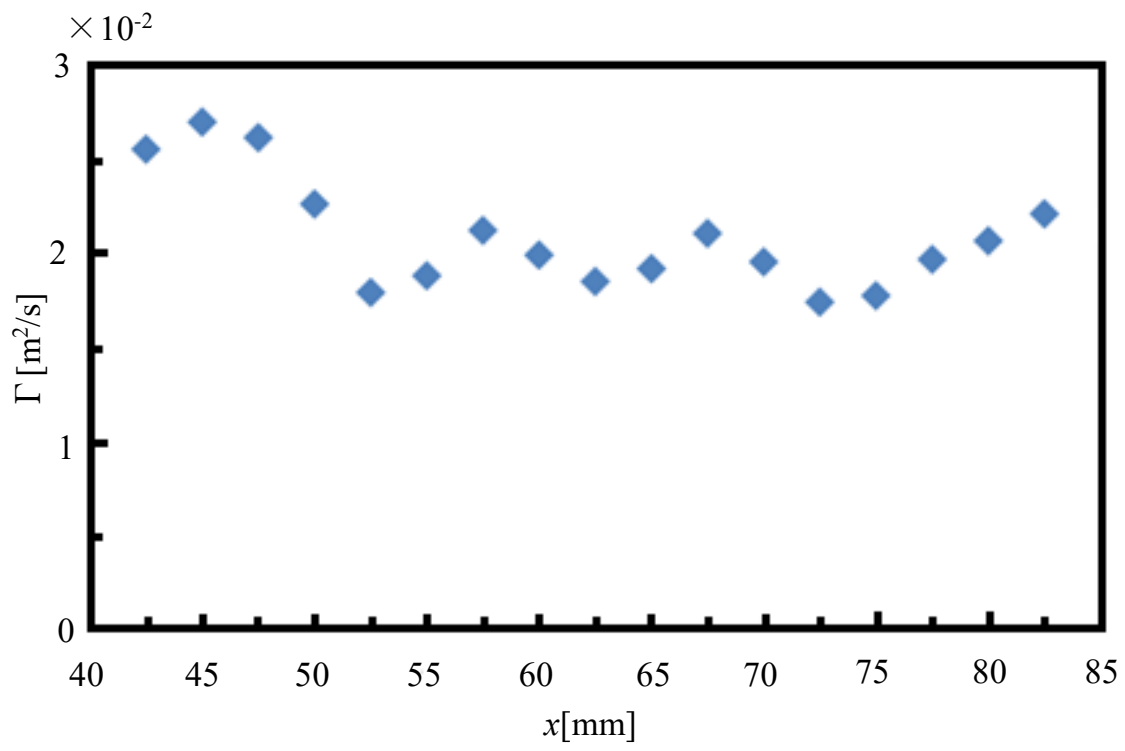

Fig. 4.1 Variation of circulation along $x$. Circulation rapidly decrease from $x=45$ to $55 \mathrm{~mm}$ then stays nearly constant. This implies that vorticity is dissipated significantly in the range around $x=45$ to $55 \mathrm{~mm}$.

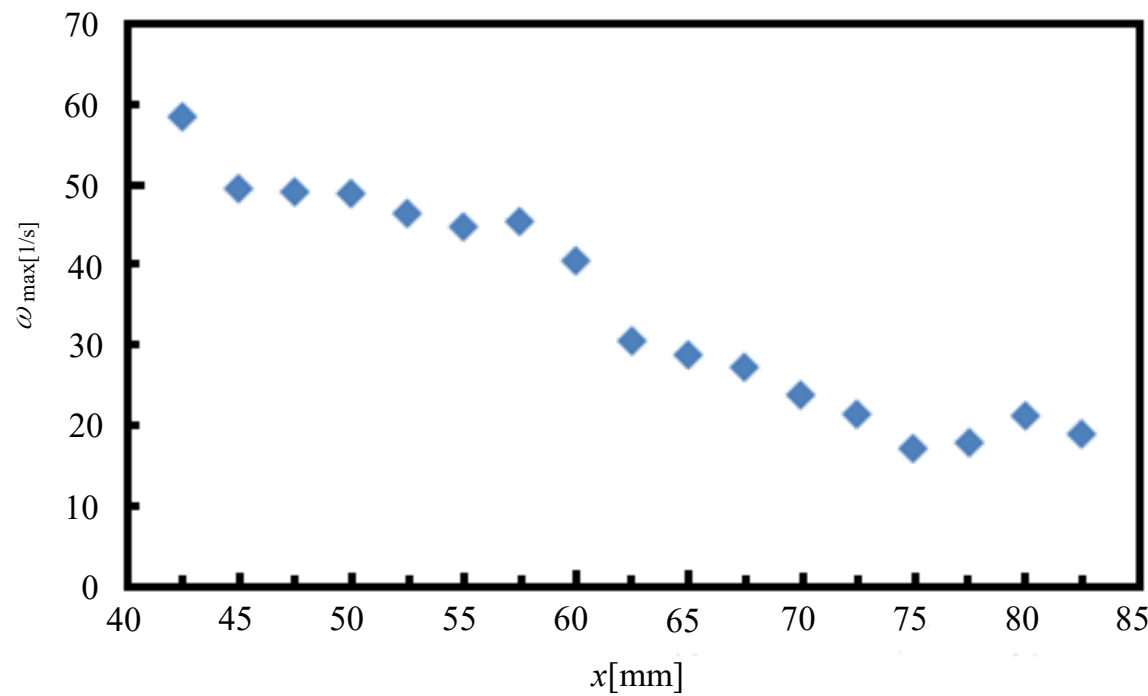

Fig. 4.2 Variation of maximum vorticity along $x$. The maximum vorticity gradually decreases from $x=40$ to $60 \mathrm{~mm}$, then rapidly decreases toward $x=80 \mathrm{~mm}$. This implies that vorticity diffusion to surrounding fluid is moderate in highly interacting stage, and it is significant in weakly interacting latter stage.

\subsection{Time variation of vorticity distribution}

Time (phase) variation of vorticity distribution is presented in Fig.4.3. At the phase 10/60, center of the leading vortex of two pairing vortices comes to about $x=48 \mathrm{~mm}$, and afterwards the pairing process with the following vortex until phase $60 / 60$ is continued. Since the separated shear layer grows with entrain of surrounding irrotational flow, it is seen that there is almost no vorticity except in the vicinity of the vortices. Although the leading vortex shows round shape at the phase 10/60, in later phases it is stretched in the flow direction with gradual decrease of maximum vorticity at the center due to moderate vorticity diffusion, then after phase 21/60 the maximum vorticity is rapidly decreases due to rapid vorticity diffusion. Around phase 21/60, center of the vortex is located at around $x=60 \mathrm{~mm}$ and keep moving toward downstream, and their location is corresponding to the starting location of rapidly decrease of maximum vorticity shown in Fig.4.2. The following vortex also shows round shape at phase 25/60, and 

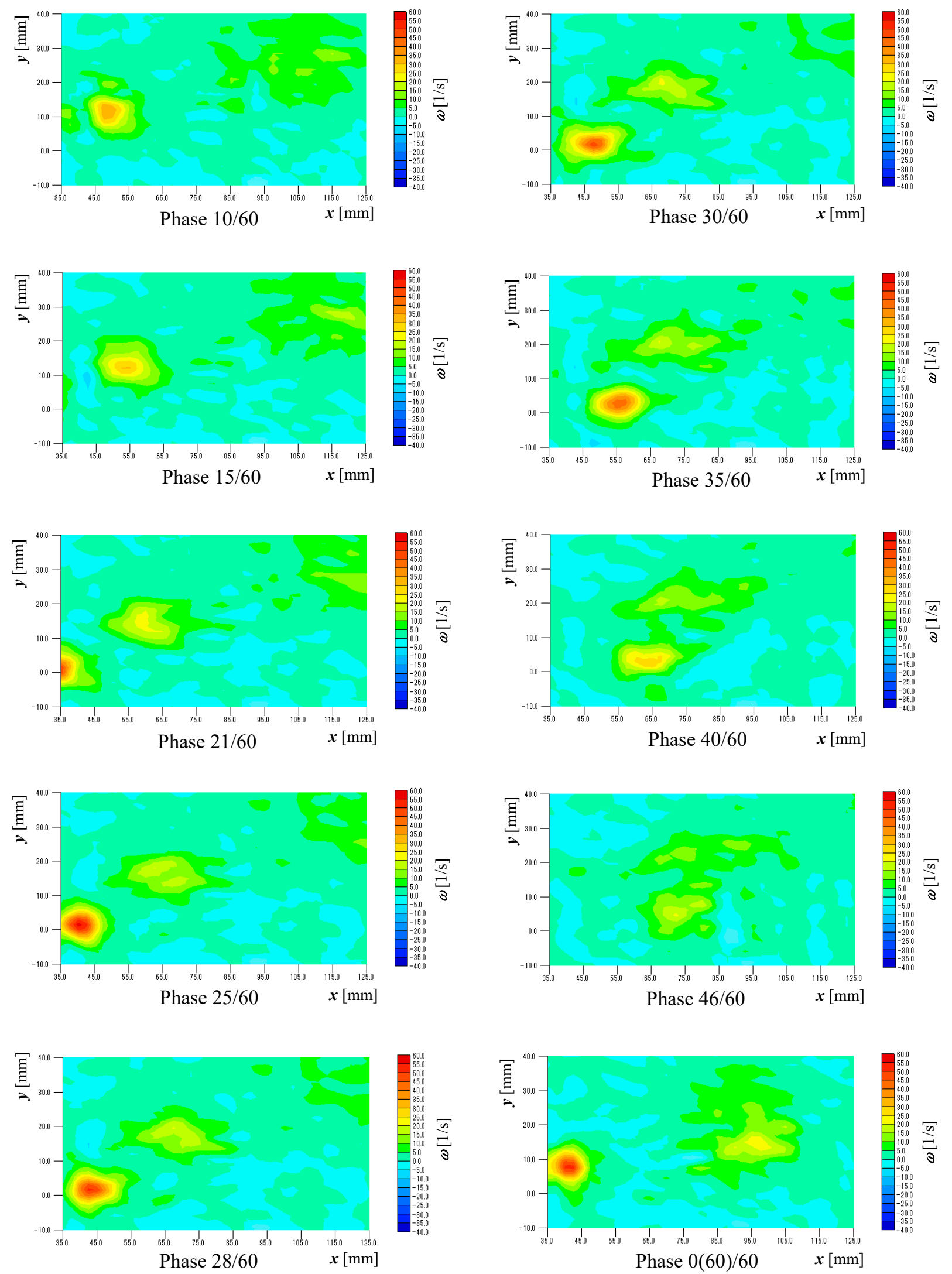

Fig. 4.3 Variation of vorticity distribution with phase. There is almost no vorticity except in the vicinity of the vortices. After the phase 10/60, the leading vortex is stretched with gradual decrease of maximum vorticity. After phase 21/60 the maximum vorticity is rapidly decreased. Similar things are happening on the following vortex from the phase $25 / 60$. 
then it is stretched in the flow direction with gradual decrease of maximum vorticity due to moderate vorticity diffusion. After that, as one can see from phases $40 / 60$ and $46 / 60$ of the figure, the maximum vorticity is rapidly decreased from about $x=60 \mathrm{~mm}$ by rapid diffusion of vorticity to the surrounding irrotational flow. This means the leading vortex in which vorticity diffusion is somewhat progressed is stretched in flow direction by interaction with the following vortex, and then the following vortex in which vorticity diffusion is somewhat progressed is also stretched in flow direction. Therefore, after each vortex stretching, vorticity diffusion to the surrounding flow becomes significant. At phase $46 / 60$, it is seen that the two pairing vortices are aligned vertically, and the vortices is considered to came to the merging location defined by Ho \& Huang (1982) where sub-harmonics must reach its peak.
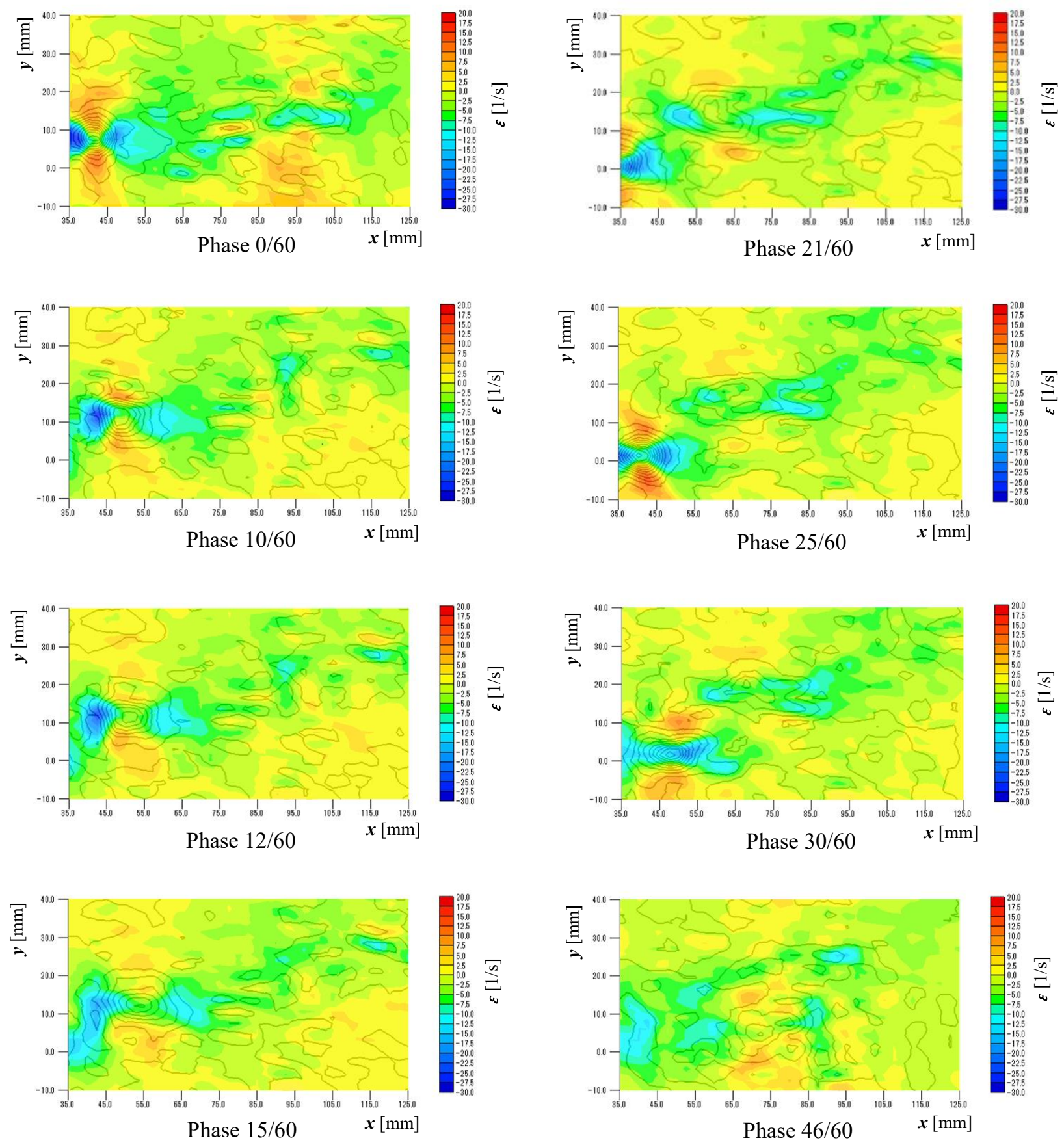

Fig. 4.4 Variation of shear strain rate distribution with phase superimposed on vorticity contours. At the phases $10 / 60$ and 12/60, the leading vortex is stretching and strong shear strain is appearing at upstream edge of the leading vortex. At the phase 15/60, the leading vortex is fairly stretched. Similar things are happening on the following vortex from the phase 25/60. At the merging location, shear strain become fairly weak. 


\subsection{Time variation of shear strain rate distribution}

Time (phase) variation of shear strain rate is presented in Fig.4.4 of previous page. Where contour lines of the vorticity distribution were superimposed as thin solid lines for better clarity of positional relation with shear strain rate distribution. Although entire curved separated shear layer is considered to be deformed by shear strain in general sense, there is almost no shear strain except in the vicinity of the vortices. At the phases 10/60 and 12/60, the leading vortex is in the stage of stretching caused by interaction with the following vortex (hidden in the left hand side), and hence strong shear strain is appearing at upstream edge of the leading vortex. At the phase 15/60, the leading vortex is fairly stretched and no further stretching is seen up to phase 25/60. Therefore, in this period, the shear strain at the upstream edge of the leading vortex is subsided. On the other hand, the following vortex is starting to be starched due to interaction with the leading vortex from about phase $25 / 60$, and strong shear strain is seen at the downstream edge of the following vortex.

At about this phase, the following vortex was just formed by rolling up of the separated shear layer, and another strong shear strain is also appeared at the upstream edge of the following vortex. Similar thing is happening at the upstream edge of the leading vortex at the phase 0/60. At the phase 30/60, when the following vortex is already fully stretched, although slight shear strain is remained at the downstream edge of the following vortex, deformation of the fluid is almost ended. At the phase 46/60, when the two vortices are aligned vertically and they reached at the merging location, shear strain become fairly weak. Therefore, in the early stage of the pairing, strong shear strain is appeared due to stretching of the vortices and hence energy of the vorticity would be dissipated indirectly.
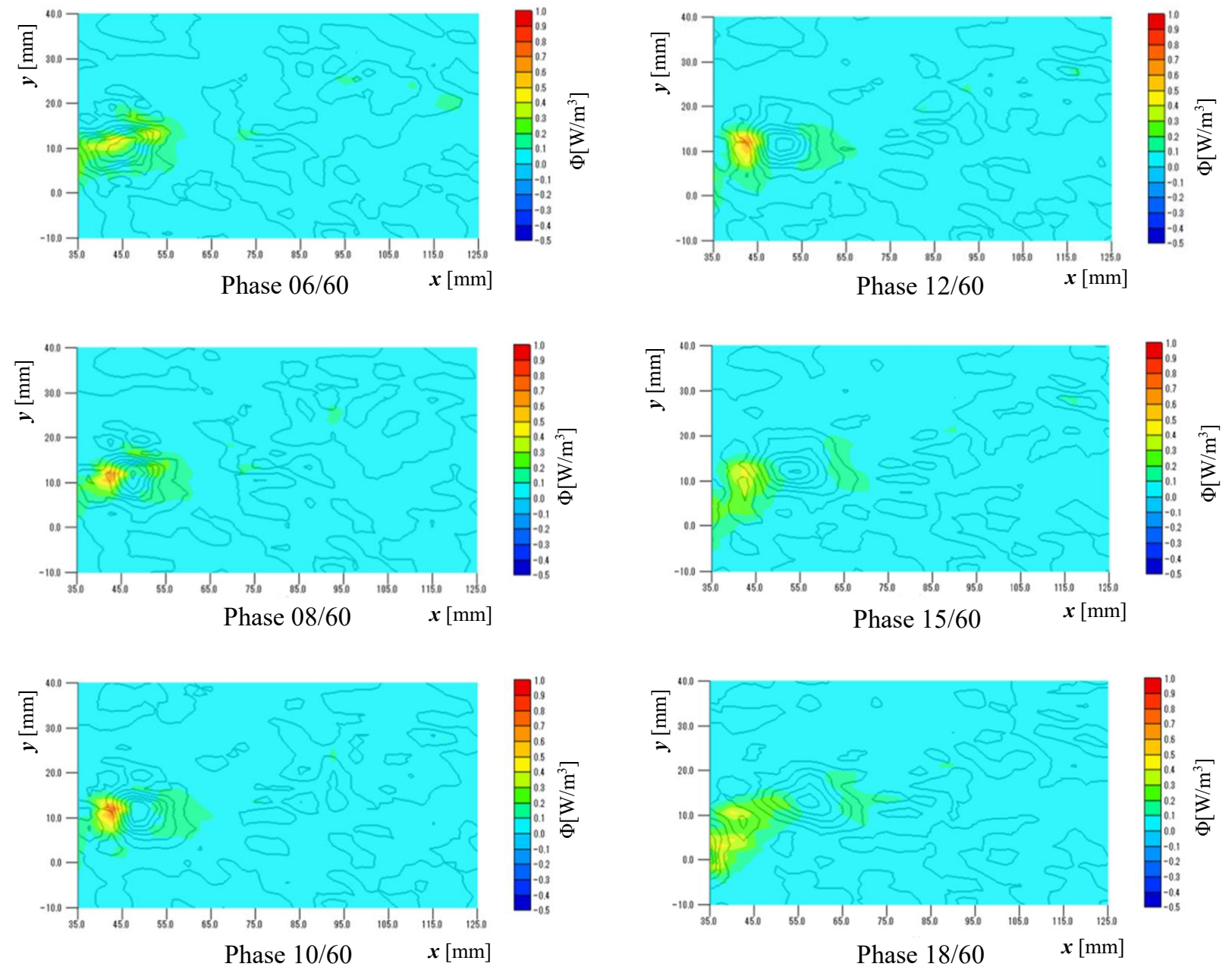

Fig. 4.5 Variation of dissipation function distribution with phase. Mainly variation of the leading vortex at early phase of vortex pairing is shown here. As seen from the phases 8/60 to 12/60, strong dissipation is seen when the leading vortex is stared to be stretched by the interaction with the following vortex. 


\subsection{Time variation of dissipation function distribution}

Time variation of dissipation function distribution is presented in Figs.4.5 of previous page and 4.6 of this page. Where contour lines of the vorticity distribution were superimposed as thin solid lines for better clarity of positional relation with dissipation function distribution. Figure 4.5 is mainly showing behavior of the leading vortex, and Fig.4.6 is mainly showing behavior of the following vortex. At phase 6/60 of Fig.4.5, leading vortex was just detached from the separated shear layer and it is slightly stretched in flow direction but the value of the dissipation function is still low. At phase 6/60 in Fig.4.4 of shear strain rate distribution, strong shear strain was observed at the upstream edge of the leading vortex. Therefore, it is not always true that the dissipation function shows high value in the region of strong shear strain. Then, from phases 8/60 to 12/60 in Fig.4.5, high value of dissipation function is seen at the upstream edge of the leading vortex, and it is subsided by phase $15 / 60$. From the fact that the shape of the vortex presented by vorticity distribution is still round from phase $8 / 60$ to $10 / 60$, energy dissipation becomes significant when the leading vortex is started to be stretched by interaction with following vortex out of view flame in the left side. By the time the vortex is obviously stretched, energy dissipation would be already ended.

At phase 21/60 of Fig.4.6, the following vortex is just appeared in the view flame, and region of large dissipation function is appearing at the downstream edge of the following vortex until phase 30/60. In this period, strong shear strain is appearing at downstream part of the following vortex, and hence the following vortex would also exhibits strong energy dissipation with shear strain in the process of stretching caused by interaction with the leading vortex. From these facts, although the rotational flow motion is not directly involved in the behavior of the dissipation function $\Phi$, rotational motions of the vortices in the pairing process cause shear strain and consume their own rotational energy which means decrease of vorticity. It is also interesting to note that there is almost no energy dissipation at the center
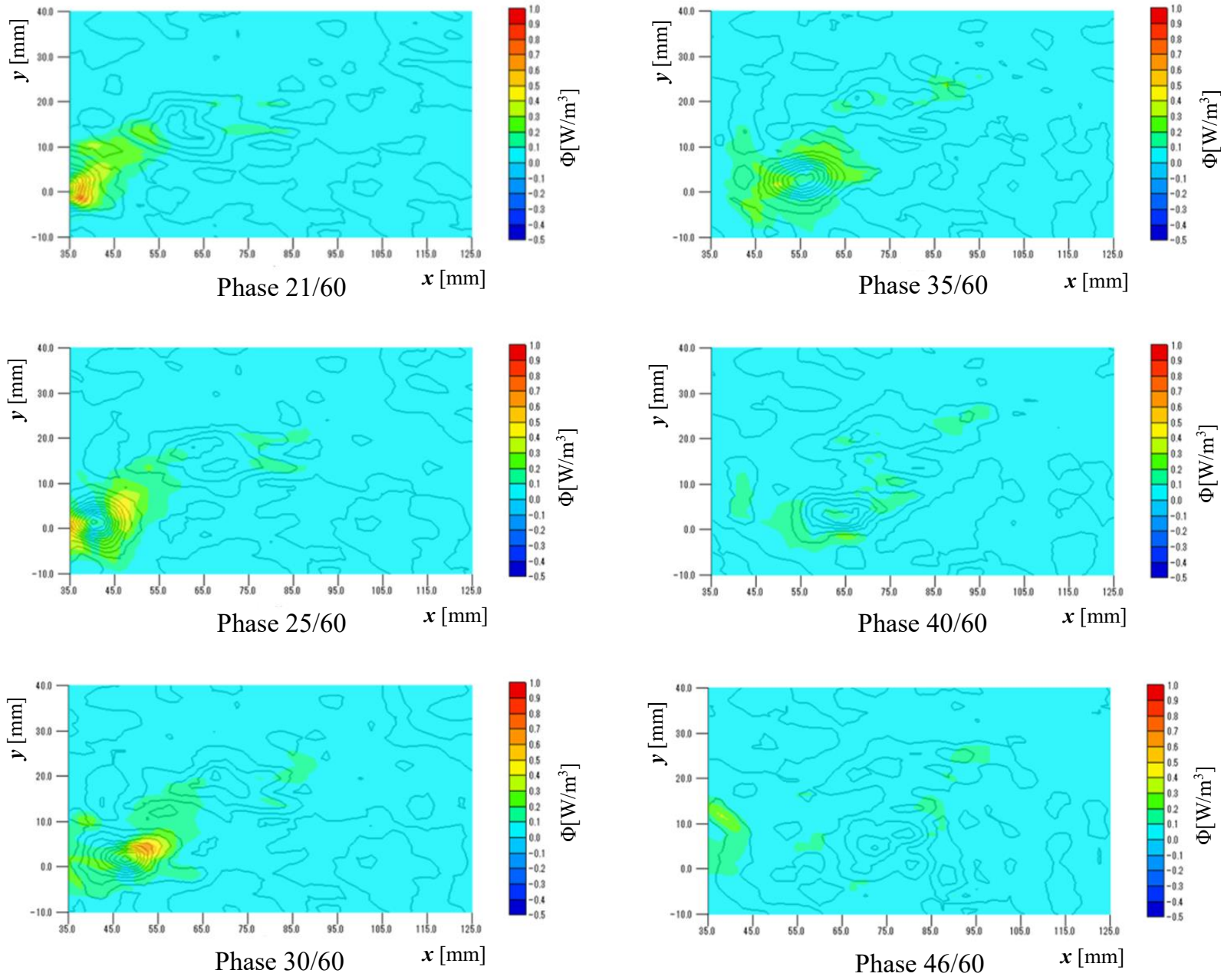

Fig. 4.6 Variation of dissipation function distribution with phase. Mainly variation of the following vortex at later phase of vortex pairing is shown here. As seen from the phases 21/60 to 30/60, strong dissipation is seen when the following vortex is stared to be stretched by the interaction with the leading vortex. 
of the vortices. After phase 35/60, values of the dissipation function rapidly subsided. In particular, at about phase 40/60, two vortices are aligned vertically with respect to flowing direction which is corresponding to the "merging location" defined by Ho \& Huang (1982), and this fact is implying that the interaction between two merging vortices are ended and dynamic vortex pairing is completed at this location.

\section{Conclusions}

Vortex pairing process in a curved shear layer was investigated by means of L.D.V. and phase ensemble averaging technique and following results are obtained:

(1) Vorticity diffusion from vortices to surrounding fluid is moderate in the highly interacting stage of vortex pairing with stretching, but it becomes significant in the weakly interacting fully stretched latter stage.

(2) Since the separated shear layer grows with entrain of surrounding irrotational flow, there is almost no vorticity except in the vicinity of the vortices.

(3) Although entire curved separated shear layer is considered to be deformed by shear strain in general sense, there is almost no shear strain except in the vicinity of the vortices. But time averaged view of the curved shear layer shows wide spread of shear strain.

(4) Energy dissipation becomes significant when a vortex is started to be stretched by interaction with neighboring vortex, but by the time the vortex is obviously stretched, energy dissipation was already ended.

(5) When the two vortices are aligned vertically with respect to flowing direction which is corresponding to the "merging location", interaction between pairing vortices are ended and dynamic vortex pairing is completed.

(6) Although the rotational flow motion is not directly involved in the behavior of the dissipation function $\Phi$, rotational motions of the vortices in the pairing process cause shear strain and dissipate their own rotational energy.

\section{References}

Aref, H. and Siggia, E.D., Vortex dynamics of the two-dimensional turbulent shear layer, Journal of Fluid Mechanics, Vol.100, Issue 4 (1980), pp.705- 737.

Browand, F.K. and Weidman, P.D., Large scales in the developing mixing layer, Journal of Fluid Mechanics, Vol.76, Issue 1 (1976), pp.127-144.

Gerrard, J.H., The Mechanics of the Formation Region of Vortices behind Bluff Bodies, Journal of Fluid Mechanics, Vol.25, Issue 2 (1966), pp.401-413.

Haniu, H. and Ramaprian, B.R., Studies on Two-Dimensional Curved Nonbuoyant Jets in Cross Flow, Journal of Fluids Engineering, Vol.111 (1989), pp.78-86.

Ho, C.M. and Huang, L.S., Subharmonics and vortex merging in mixing layers, Journal of Fluid Mechanics, Vol.119 (1982), pp.443-473.

Mair, W.A. and Maull, D.J., Bluff Bodies and Vortex Shedding - A Report on Euromech 17, Journal of Fluid Mechanics, Vol.45, Issue 2 (1971), pp.209-224.

Metcalfe, R.W., Orszag, S.A., Brachet, M.E., Menon, S. and Riley J.J., Secondary instability of a temporally growing mixing layer, Journal of Fluid Mechanics, Vol.184 (1987), pp.207-243.

Miyakoshi, K., Haniu, H., Kim, S., Takai, K. and Islam, M.R., Decay of Vorticity in Separated Shear Layer, Journal of Fluid Science and Technology, Vol.5, No.2 (2010), pp.331-339.

Sarpkaya, T. and Schoaff, R.L., Inviscid Model of Two-Dimensional Vortex Shedding by a Circular Cylinder, AIAA Journal, Vol.17, No.11 (1979), pp.1193-1200.

Savitzky, A. and Golay, M.J.E., Smoothing and Differentiation of Data by Simplified Least Squares Procedures, Analytical Chemistry, Vol. 36, No.8 (1964), pp. 1627-1639.

White, F.M., Viscous fluid flow, McGraw-Hill Inc., ISBN 0-07-069710-8 (1974)

Wignanski, I., Oster, D., Fiedler, H. and Dziomba, B., On the perseverance of quasi-two-dimensional eddy-structure in a turbulent mixing layer, Journal of Fluid Mechanics, Vol.93, Issue 2 (1979), pp.325 - 335.

Winant, C.D. and Browand, F.K., Vortex pairing: the mechanism of turbulent mixing-layer growth at moderate Reynolds number, Journal of Fluid Mechanics, Vol.63, Issue 2 (1974), pp.237-255. 